\title{
Planes de estudio de la Facultad de Economía de la UADY (1998 y 2011)
}

\author{
Study plans of the Faculty of Economics of the UADY (1998 and 2011)
}

David de Jesús Santoyo Manzanilla*

*Licenciado en Educación por la UADY; Licenciado en Ciencias Teológicas por la Universidad Iberoamericana; Maestro en Educación Superior por la UADY. Actualmente es docente, coordinador de tutorías, coordinador de seguimiento académico y coordinador de difusión cultural de la Facultad de Economía de la UADY.

\section{ARTÍCULO DE REVISIÓN RESUMEN}

Este artículo tiene la finalidad de compartir algunas reflexiones teóricas con respecto a las modificaciones de los planes de estudio de los años 1998 y 2011 de la Facultad de Economía de la Universidad Autónoma de Yucatán (UADY), que se llevaron a cabo en los años respectivos ya mencionados $(1998,2011)$ para cumplir con las exigencias de un mundo globalizado rápidamente cambiante. Muestra cómo los cambios que suscitaron tales modificaciones fueron la obsolescencia en el modelo educativo y en las exigencias laborales. El artículo se divide en cuatro apartados que, apoyados por una revisión de la literatura, exponen las siguientes cuestiones: primeramente, se plasma el contexto educativo de nivel superior del Estado de Yucatán en el campo de la Economía; se explica cómo nace la Escuela de Economía de la UADY; se describen las razones para la modificación del plan de estudios en 1998; se justifica la modificación del plan de estudios 2011; y se discute la viabilidad de que el plan de estudios reformado (2011) logre un mejor impacto social y laboral para los egresados. Concluye este trabajo enfatizando la necesidad de revisiones y cambios continuos en los planes de estudio que tengan como base estudios de seguimiento a egresados y empleadores y cuya concepción del modelo educativo que se aplique esté basado en la interdisciplinariedad y en los últimos enfoques y teorías educativas acordes con los tiempos postmodernistas en que vivimos.

Palabras clave: Planeación de programas, planeación educativa, planeación universitaria, planes de estudio, políticas educativas.

\section{ABSTRACT}

This article has the purpose of sharing some theoretical reflections regarding the modifications of the curricula of the years 1998 and 2011 of the Faculty of Economics of the Autonomous University of Yucatán (UADY), which took place in the years respective aforementioned $(1998,2011)$ to meet the demands of a rapidly changing globalized world. It shows how the changes that caused such modifications were the obsolescence of the educational model and the work demands. The article is divided into four sections which, supported by a review of the literature, expose the following questions: firstly, the higher level educational context of the state of Yucatán in the field of the economy is embodied; it explains how the School of Economics of the UADY was born; the reasons for the modification of the curriculum are described; amending curriculum 2011 is justified; and the feasibility of reformed curriculum (2011) To achieve a better social and labour impact for graduates is discussed. This work concludes by emphasizing the need for continuous reviews and changes in curricula based on follow-up studies to graduates and employers, and whose conception of the educational model that is applied is interdisciplinarity and in the latest approaches and educational theories in line with the postmodern times in which we live.

Keywords: program planning, educational planning, university planning, study plans, educational policies.

\section{INTRODUCCIÓN}

Desde la segunda guerra mundial, ha habido en todos los países la necesidad de una reforma de la enseñanza (UNESCO, s.f, p. 5); por ello, hasta el día de hoy, muchos países han tratado de buscar la mejor manera de preparar a los estudiantes en su carrera profesional.

Un plan de estudios de calidad, en palabras de Stabback (2016, p. 6), debe presentar una selección consciente y sistemática de 
Perspectivas Docentes 64

Planes de estudio de la Facultad de Economía de la UADY (1998 y 2011)

Study plans of the Faculty of Economics of the UADY (1998 and 2011)

David de Jesús Santoyo Manzanilla

conocimientos, capacidades y valores; debe tomar en cuenta cuestiones como qué, por qué, cuándo y cómo deberían aprender los estudiantes.

Todo plan debe contar con un programa de estudios que tome en cuenta la manera en que los jóvenes aprenden y sus propias necesidades; ser lo suficientemente flexible para adaptarse a través del tiempo y, por supuesto, a las circunstancias locales (Stabback, 2016, p. 28).

La educación contemporánea está intentando cambiar la pasividad estudiantil para volverla dinámica y activa. En los planteles de niveles superiores esto ha sido un gran paso, los futuros profesionistas ya tienen la oportunidad de egresar no solo con conocimiento teórico sino también práctico. Esto trae consigo más oportunidades para los estudiantes pues obtendrán experiencia suficiente para ser competentes en el ámbito laboral en el que se encuentren inmersos.

Las instituciones educativas deben procurar la trascendencia misma de la educación y generar impactos sociales con un cambio de mentalidad en sus estudiantes para que estos puedan ser analíticos y críticos ante las diversas y complejas situaciones actuales. Se espera que un profesional además de ser competente en su campo de estudio, adquiera valores que conlleven beneficios ante la sociedad y que sea promotor de las buenas acciones.

El objetivo del presente trabajo es presentar las razones que motivaron la modificación de los últimos dos planes de estudio de la Facultad de Economía de la UADY, es decir, el de 1998 y 2011, con el fin de sustentar las fortalezas y debilidades que ambos planes han presentado durante su implementación en la vida profesional y social de los estudiantes. Para entrar en contexto, el primer apartado hace referencia histórica a la institución; después se justifica la existencia de una Escuela de Economía como tal en el Estado de Yucatán; se analizan los dos planes de estudio (1998 y 2011), y se argumenta porqué la modificación del plan 2011 es más promisoria social y laboralmente para los egresados.

\section{NACE LA ESCUELA DE ECONOMÍA DE LA UADY}

El seis de diciembre de 1971 inicia sus cursos la Escuela de Economía de la Universidad de Yucatán. Antes de ello, el rector de aquel entonces de la Máxima Casa de Estudios, el Dr. Alberto Rosado G. Cantón, había presentado al H. Consejo
Universitario, en la sesión celebrada el día 29 de julio de 1971 , la propuesta de la creación de dicha escuela; pero fue hasta dos meses más tarde cuando es aprobada, es decir, el día cinco de octubre. Desde entonces la Escuela de Economía se ha regido con la misión de formar profesionales e investigadores en las ciencias económicas y políticas públicas, con valores éticos y capacidad crítica que contribuya al desarrollo sustentable de la región (Facultad de Economía, UADY, 2017).

Fueron 19 profesionistas los que conformaron la primera e ilustre planta docente de esa noble dependencia, algunos de ellos formados en la UNAM y en el Instituto Tecnológico y de estudios Superiores de Monterrey; fueron 60 los alumnos, siendo su primer director el Lic. Eduardo Rodríguez Pérez. En tan sólo dos años, la Escuela de Economía se traslada al edificio de la histórica calle 61 número 550 del centro de la ciudad de Mérida, donde actualmente se encuentra la Unidad de Ciencias Sociales del Centro de Investigaciones Regionales "Dr. Hideyo Noguchi" de la Universidad Autónoma de Yucatán (UADY).

Posteriormente, en la década de 1980, se muda al oriente de la ciudad y durante sus veintinueve años cambia en cuatro ocasiones sus planes de estudio. Es para septiembre de 2013 cuando la Facultad de Economía se establece en donde hasta el día de hoy permanece erguida y consolidada, el Campus de Ciencias Sociales, Económicas-Administrativas y Humanidades (Facultad de Economía, UADY, 2017).

\section{¿POR QUÉ UNA ESCUELA DE ECONOMÍA EN YUCATÁN?}

No existía en el Estado y región un programa de estudios que proporcionara al estudiante de esta disciplina un marco conceptual y metodológico apropiado para el análisis y formulación de políticas fundamentadas en información económica, política y social relevante, localmente hablando.

La economía del Estado y sus programas de desarrollo económico hasta antes de que egresara la primera generación de esta escuela eran elaborados a priori, es decir, se intentaba dirigir la política económica de Yucatán limitándose a imitar teorías políticas, ideologías e instituciones que habían funcionado más o menos bien en otros estados pero sin considerar su impacto sobre los resultados económicos locales, con una geografía, recursos y modus vivendi radicalmente diferente de los del resto de la República. 
Perspectivas Docentes 64

Planes de estudio de la Facultad de Economía de la UADY (1998 y 2011)

Study plans of the Faculty of Economics of the UADY (1998 and 2011)

David de Jesús Santoyo Manzanilla

Las políticas económicas se caracterizaban por la excesiva simplificación del análisis económico, que realiza un análisis cuantitativo de los problemas en forma abstracta, sin considerar el marco social y político del que emanan. Pero a partir de 1976 las cosas cambian. Había ya en el Estado un grupo pequeño de egresados preparado cabalmente en esta área. La reflexión, la crítica, el apoyo y las propuestas económicas hechas por ellos en los medios y en las dependencias públicas y/o privadas a fin de construir un sistema económico apropiado se hizo una realidad.

El papel de estos profesionales economistas era adecuar los recursos del Estado de Yucatán, a veces escasos, a los fines que le son múltiples; fines ante los cuales el economista no debe permanecer neutral, sino que debe participar activamente en su determinación, para que quienes toman decisiones de política hoy día en el Estado y región reconozcan la existencia de costos de oportunidad, y distingan fines auténticos de medios que podrían parecer fines.

Llegada la década de los 80, Reyes (2007) menciona que debido a la prolongada recesión económica que agravaba la deuda externa, se aligeraron procesos de ajuste en las naciones latinoamericanas donde se había registrado una importante reducción en el PIB y altos niveles de inflación, produciendo un descenso de los indicadores económicos y sociales. No obstante, Yucatán con su escuela de economía de reciente creación para ese entonces, ya ponía su granito de arena en la formación de economistas con una visión generadora de conocimientos pertinentes y relevantes hacia la aplicación y búsqueda de soluciones a los problemas económicos y al desarrollo sustentable de la región sur -sureste (Facultad Economía, UADY, 2017).

\section{PLAN DE ESTUDIOS 1998}

Tanto los once directores que la Escuela de Economía (desde septiembre de 1984, Facultad de Economía de la Universidad Autónoma de Yucatán) como las 42 generaciones que han egresado de esta Facultad desde hace más o menos cuatro décadas han tenido una permanente actitud de renovación que ha caracterizado siempre esta dependencia. Como resultado de ello, se han hecho sucesivas reformas al plan de estudios de la Licenciatura en Economía en los siguientes años: 1976, 1982, 1990, 1992, 1998 y la del 2011 que propone un perfil de egreso basado en competencias nacionales e internacionales.
La modificación de 1982 trajo consigo un cambio de currículum en el que se pretendía generar profesionales en el ámbito productivo, macro y microeconómico, particularmente se buscaba contribuir a la transformación con carácter público y social. En 1990, por otro lado, se modificó el plan de estudio con respecto a la búsqueda del mejoramiento académico y la vinculación con los sectores productivos. Finalmente, en 1992, se proporcionó al currículum una serie de elementos prácticos en la formación que permitieran un mayor papel en el ámbito privado por lo que el número de asignaturas prácticas fue aumentando. Dicho plan pretendía proporcionar al economista local una serie de instrumentos que le permitieran hacer más aplicativa la carrera (pp. 1-17).

Entonces, ¿Por qué comenzar el análisis de la trascendencia social que conlleva la revisión de los planes de estudio de la Facultad de Economía con el plan de estudios de 1998 cuando antes se habían llevado a cabo cuatro revisiones? Entre las razones que pudiesen argüirse están las siguientes:

- $\quad$ Fue un plan de estudios acreditado por los Comités Interinstitucionales para la Evaluación de la Educación Superior (por sus siglas, CIEES) y por lo tanto representa un excelente marco de referencia para iniciar con él esta reflexión.

- Representa el quinto esfuerzo sistemático de responder pertinentemente a las necesidades socioeconómicas del entorno y la modernidad, retomando los aciertos y eliminando los errores que pudieron haber tenido los planes anteriores.

- Es el plan vigente que operó en la Facultad hasta el 2011, es decir, es el cimiento curricular sobre el cual se harían las próximas revisiones.

La propuesta de revisión del plan de estudios llevada a cabo en 1998, siendo director de la Facultad el Lic. Jorge Luis Canché Escamilla, apuntaba a dar atención a las recomendaciones de los CIEES: fortalecer el enfoque microeconómico y así compensar el antiguo desbalance del plan anterior que favorecía la macroeconomía. Además, el área de métodos cuantitativos se orientó con mayor claridad a la resolución de problemas de índole microeconómico, en consonancia con la mayoría de los problemas económicos de la región, las necesidades sociales y demanda de profesionales del ramo. 
Perspectivas Docentes 64

Planes de estudio de la Facultad de Economía de la UADY (1998 y 2011)

Study plans of the Faculty of Economics of the UADY (1998 and 2011)

David de Jesús Santoyo Manzanilla

El nuevo plan fue ampliamente discutido y analizado en el interior de la Facultad, con profesores de la planta académica, en lo individual y por áreas, así como con los alumnos que conforman el Consejo Académico en particular, y desde luego, en el exterior con los egresados, asociaciones de profesionales y empleadores principalmente.

El resultado fue un plan de estudios conformado por 47 asignaturas y cuatro seminarios para un total de 51 , todos obligatorios, distribuidos en nueve semestres con un total de siete áreas las cuales son: Teoría Económica, Métodos Cuantitativos, Historia Económica, Contables e Instrumentales, Económicas Complementarias, Investigación Económicas e Integración profesional. En conjunto, se busca cubrir las áreas básicas del alumno brindándoles suficientes herramientas para que conozcan su papel en el campo profesional.

Indiscutiblemente siempre se han requerido profesionales capacitados que contribuyan al desarrollo económico y social de México y particularmente de Yucatán, pero para ello es sumamente necesario que el economista se encuentre trabajando directamente en los agentes que conforman esta estructura económica. Un economista debe ser poseedor de un carácter social a fin de procurar mejorar los niveles de vida del ser humano entendiendo la realidad objetiva de la economía que implica generar renovaciones constantes de conocimiento (pp. 36 y 38 ).

Estas aptitudes de manera integral, dieron paso a la justificación del cambio de sentido del perfil de egreso, de lo macro a lo microeconómico, pues se fundamentaba en el hecho de que los logros y fracasos económicos experimentados en la década de los noventa dejaron una enseñanza muy clara: es importante que todos los agentes económicos (no sólo el gobierno, sino también las empresas y los consumidores) cuenten con el apoyo de gente capacitada para analizar los fenómenos económicos y para proponer acciones que minimicen el impacto de las crisis e incrementen el potencial de las expansiones. Los problemas que aquejan a la población han de atenderse, entonces, considerando las relaciones sociales, económicas y políticas que imponen las nuevas estructuras que están en la última fase de su consolidación.

Era ya imperativa la necesidad de establecer dentro de un nuevo currículum líneas de estudio que integren los contenidos de los cursos a través de toda la carrera, de manera que deriven en áreas específicas de actividad en las cuales el egresado pueda desempeñarse profesionalmente. Estas áreas ya están plenamente identificadas: el sector público y privado nacional, el ámbito internacional, y la investigación económica. Debía tomarse en cuenta la posibilidad de brindar al alumno las habilidades suficientes para colocarse en un puesto que no sólo sea acorde al ámbito de competencia, sino que sirva, al mismo tiempo, de complemento en la formación. Así, el Nuevo Plan de Estudios (1998) presenta su propósito fundamental:

Formar un profesional capaz de ubicarse como analista y organizador de los procesos económicos tanto de empresas privadas como de órganos de gobierno, con miras a contribuir al desarrollo de la sociedad mexicana ubicada en el contexto de la globalización económica. Para ello, el economista debe emplear tanto el bagaje de conocimientos como el herramental disponible, procurando hacer más eficientes las estructuras a su cargo, empleando adecuadamente los recursos disponibles y alcanzando las más altas metas económicas posibles, independientemente del nivel decisorio en el que se encuentre y atendiendo en todo momento a los principios básicos de la conducta profesional. (P.39)

No obstante y a pesar de todas las oportunas modificaciones de contenido del plan 1998, había una debilidad pedagógica que a largo plazo iba a repercutir en la capacidad del egresado de identificarse con el mercado laboral real al que estaba dirigida su formación: se seguía proponiendo una metodología de enseñanza tradicional, consistente en la exposición oral de los temas por parte del profesor, apoyándose en recursos audiovisuales, y en ciertos casos, se daba énfasis a la realización de trabajos de investigación. Esto repercutía en varios aspectos:

- La formación profesional del economista seguía siendo abstracta pues rara vez correspondía la enseñanza en el aula de los cursos con la realidad económica de la localidad y/o región.

- No había una eficiencia funcional del egresado con los puestos posibles de ser ocupados por un economista pues su formación era buena en contenidos, pero distante de la práctica vivencial.

- No lograban aglutinar su formación académica para hacer frente a los problemas particulares de índole económica que tenían que enfrentar en los distintos ámbitos que su trabajo les exigiese. 
Perspectivas Docentes 64

Planes de estudio de la Facultad de Economía de la UADY (1998 y 2011)

Study plans of the Faculty of Economics of the UADY (1998 and 2011)

David de Jesús Santoyo Manzanilla

En conclusión, no sólo el cambio drástico de los esquemas internacionales de economía y comercio que repercuten hoy día profundamente sobre todos los rincones de la tierra (intensa recesión, neoliberalismo, globalización, neocapitalismo, comercio en línea, extrema marginación, destrucción medioambiental, privatización del sector público, etc.) hicieron obsoleto con prontitud el plan de estudios 1998, sino que el modo de enseñar, es decir, el modelo académico conductista quedó rebasado por una realidad de dimensiones gigantescas imposible de ser abordado por la vieja teoría educativa de los reforzamientos.

\section{PLAN DE ESTUDIOS 2011}

Era necesario un cambio, un diseño curricular que tomara en cuenta la realidad operativa de los profesionistas económicos tanto como la forma de transmisión o mejor dicho construcción del conocimiento. Es bajo esta premisa que surge con denuedo por parte del entonces director de la Facultad, el Dr. Alberto Quintal Palomo, la renovación integral del plan de estudios a principios del año 2010 y como una condición imperativa para que la Facultad siguiera consolidándose como una dependencia universitaria formadora de egresados de calidad.

Es por ello que la propuesta de modificación del Plan de Estudios de Licenciado en Economía del 2011 se fundamenta básicamente en el Modelo Educativo y Académico (MEyA) de la UADY, enfocado en el desarrollo de competencias profesionales. Se pretende como un medio para lograr esto innovar las prácticas educativas e implementar un currículo flexible, que modifique el papel del docente, convirtiéndolo en un facilitador del conocimiento, lo cual además, reduzca la presencialidad, enfocando el desarrollo de las sesiones de clase a la realización de actividades fuera del aula, permitiendo que se implementen nuevas modalidades educativas, como la semipresencial y la educación a distancia.

Este plan es de tipo flexible y consta de nueve semestres y el mínimo de créditos que deben cursar los estudiantes por semestre es de 19. Las asignaturas están divididas en siete áreas académicas: Teoría Económica, Métodos Cuantitativos, Historia Económica, Contables e Instrumentales, Económicas Complementarias, Investigación Económicas y Política Económica. Se detectó la necesidad de incluir esta nueva área debido a que la formulación de estrategias principalmente en instituciones gubernamentales, las cuales conducen la economía del país, son parte esencial de un economista competente. Esta última reemplaza al área de Integración profesional del plan de estudio de 1998 pues en realidad no era un área académica sino una actividad compleja resultante de vincular lo teórico-práctico.

La evaluación de las acciones del gobierno en la economía local, regional y nacional desde una perspectiva analítica de las tendencias económicas internacionales, hace de este plan de estudio un proyecto que toma en cuenta los cambios a los que esta ciencia se enfrenta pues evoluciona de una manera acelerada en todos los niveles. Entonces, del papel desempeñado por los economistas dependerá el desarrollo de los pueblos en un sentido integral e interdisciplinario (p. 2).

Son 39 asignaturas obligatorias, es decir, 12 menos que las asignaturas y seminarios obligatorios del plan anterior. En total se cubren 241 créditos o un 70\%, mientras que las optativas y las libres representan un $20 \%$ y un $10 \%$ respectivamente dentro del Modelo Académico de la UADY. Este plan se ha comparado con otros afines en Instituciones Nacionales públicas y privadas tales como la Universidad Nacional Autónoma de México (UNAM), el Instituto Politécnico Nacional (IPN), Instituto Tecnológico Autónomo de México (ITAM), Universidad Autónoma de Campeche (UNACAM), la Universidad de Quintana Roo (UQROO), entre otros (pp. 33 y 34)

Guilar (2009, p.237) señala que "el aprendizaje es un proceso activo, de asociación, construcción y representación". El MEyA está enfocado a la resolución de problemas relacionados con la vida cotidiana de los estudiantes, todo esto, desde un enfoque constructivista y apoyándose en un desarrollo curricular en espiral tal como Bruner (1963) citado por Santrock (2003) lo define. "Se basa en pasos sucesivos por un mismo dominio de conocimiento y tiene el objetivo de promover el aprendizaje" (Bruner, 1988, p. 247). Se logra al profundizar los contenidos que se estudian en los semestres iniciales en todo el desarrollo de la propuesta curricular. Su objetivo es el siguiente:

Formar profesionales con una sólida preparación teórica y práctica y con un espíritu crítico de las principales corrientes de pensamiento económico, competente para desarrollar labores de gestión, solución de problemas y toma de decisiones para la optimización de recursos en el sector público, privado o social, en un contexto globalizado con un compromiso con el desarrollo sustentable. (p. 97)

Así el propósito de la carrera da un cambio, no solo por la formación de nuevos profesionistas a nivel teórico y práctico 
Perspectivas Docentes 64

Planes de estudio de la Facultad de Economía de la UADY (1998 y 2011)

Study plans of the Faculty of Economics of the UADY (1998 and 2011)

David de Jesús Santoyo Manzanilla

que se puedan desarrollar en distintas labores y en diversos sectores económicos de una manera eficiente, sino también la concientización social con respecto al concepto de desarrollo sustentable que marca un paso provechoso a la competitividad mundial en el que se busca un sano crecimiento económico en todos los ámbitos posibles de la sociedad.

De igual manera, el desarrollo sustentable se ha establecido como una competencia genérica (Competencia CG06) del Licenciado en Economía de la UADY. El profesionista debe tener la capacidad para identificar, plantear y resolver problemas donde el entorno natural y cultural deba ser considerado en la valoración de las alternativas (p. 60). Se espera con esto que los economistas procuren el bienestar natural por que va de la mano con el bienestar social. Se ha abierto un campo que puede ser aprovechado. En la actualidad muchos espacios naturales han empezado a ofrecerse como productos en su mayoría turísticos por lo que es necesario la toma de buenas decisiones con respecto a su manejo y control, en este caso económico.

La reestructuración curricular propuesta tiene las siguientes características y/o bondades generales:

- Está relacionada con las recomendaciones que organismos internacionales han formulado con el objetivo de mejorar los indicadores educativos de los países del orbe en aspectos tales como: política nacional de educación superior; objetivos y visión de la Facultad de Economía como dependencia; condiciones cambiantes de la economía mundial, nacional y regional; análisis académico de diversos planes de estudio de licenciatura de dicha disciplina en otras Universidades de México; el estudio del grado en economía realizado por la Agencia Nacional de Evaluación de la Calidad y Acreditación (ANECA); las recomendaciones de organismos nacionales vinculados a la educación y la enseñanza de la ciencia económica; las recientes aportaciones a los campos de la economía y del desarrollo y en la evaluación curricular correspondiente.

- Esta propuesta incorpora la modificación en lo referente a un plan de estudios flexible y además facilita la movilidad estudiantil, mediante convenios con otras universidades, así como con otras facultades de la UADY.
- Con base en el análisis de las necesidades de la sociedad, se reestructuraron las diversas áreas académicas del programa y las asignaturas que lo componen, ya sean obligatorias, optativas o libres, después de hacer una comparación de la propuesta con otros planes de estudio vigentes en la UADY, así como otros planes de estudio de la Licenciatura en Economía de instituciones de educación superior del país, lo propuesto por la ANECA, el proyecto Tunning y el análisis efectuado por expertos en contenido, así como la revisión de indicadores sobre la inserción laboral de economistas del Estado de Yucatán y de la República Mexicana.

\section{CONCLUSIÓN}

Las diversas actualizaciones por las que el currículo de la Facultad de Economía ha transitado a lo largo de su existencia han estado orientadas a la adaptación del mismo a las condiciones de sus entornos mediato e inmediato. No obstante, se hace indispensable la modificación sistemática del plan de estudios, no sólo de esta carrera sino de todas pues el entorno socioeconómico global actual siempre cambiante exige profesionales que sean capaces de adaptarse a circunstancias y escenarios dinámicos, que entiendan los factores y causas que originan dichos procesos y que tengan respuestas precisas y ágiles para enfrentar estas situaciones coadyuvando así a las instituciones, organizaciones.

A continuación se presenta la Tabla 1 que permite apreciar las diferencias y similitudes encontradas en ambos planes de estudio y que sirven como síntesis de este documento. Entre los datos se encuentra el objetivo particular, justificación de su creación e implementación, asignaturas y repercusiones y/o bondades. 
Perspectivas Docentes 64

Planes de estudio de la Facultad de Economía de la UADY (1998 y 2011)

Study plans of the Faculty of Economics of the UADY (1998 and 2011)

David de Jesús Santoyo Manzanilla

Tabla 1 Comparación de los planes de estudios 1998 y 2011

\begin{tabular}{lll}
\hline Campo & Plan de estudios 1998 & Plan de estudios 2011 \\
& $\begin{array}{l}\text { Profesionales analistas y organizadores en } \\
\text { ámbito público y privado que contribuyan al } \\
\text { desarrollo de la sociedad mexicana. } \\
\text { Objetivo }\end{array}$ & $\begin{array}{l}\text { Profesionales con preparación teórico-práctica, } \\
\text { competente en el sector público, privado o social } \\
\text { con compromiso de desarrollo sustentable. }\end{array}$ \\
Justificación & $\begin{array}{l}\text { dándoles suficientes herramientas para que } \\
\text { conozcan su papel en el campo profesional. }\end{array}$ & $\begin{array}{l}\text { Tomar en cuenta la realidad operativa de los pro- } \\
\text { fesionistas económicos como la construcción del }\end{array}$ \\
conocimiento mismo.
\end{tabular}

Elaboración propia con datos de ambos planes de estudio (1998 y 2010)

La nueva propuesta curricular 2011 para la Licenciatura en Economía implica la formación de un profesional que pueda ubicarse, incluso durante su proceso de formación, en ámbitos y escenarios diferentes al habitual, entendiendo al respecto la posibilidad de participar y realizar estancias de movilidad en diferentes instituciones de educación superior tanto del país como del extranjero, recibiendo conocimiento en universidades de reconocido prestigio en otros ambientes y conociendo entornos, idiosincrasias y ópticas diversas, con problemáticas diferentes a la de su medio, lo cual le servirá para ampliar su criterio y agudizar su capacidad de análisis y reflexión, así como recibir formación bajo condiciones y características diferentes, tanto culturales como institucionales, capacitando al estudiante bajo la óptica de la interdisciplinariedad, trabajo en equipo y trabajo en ambientes diversos. Sólo trabajando de esta manera con seguridad podrá alcanzarse aquella ya antigua misión de la Facultad, en la que se ha propuesto con vocación la búsqueda de la excelencia académica y la formación del mejor profesionista de la ciencia económica, cuidando darle además de los conocimientos académicos y cientíicos una formación humanística, cultural y deportiva fortaleciendo el vínculo del estudiante con su facultad e institución. 
Perspectivas Docentes 64

Planes de estudio de la Facultad de Economía de la UADY (1998 y 2011)

Study plans of the Faculty of Economics of the UADY (1998 and 2011)

David de Jesús Santoyo Manzanilla

\section{REFERENCIAS}

Bruner, J. S. (1963). El proceso de la educación. México: UTEHA.

Bruner, J. S. (1988). Desarrollo educativo y educación. Madrid: Morata

Facultad de Economía, UADY (1998). Nuevo Plan de Estudios de la Licenciatura en Economía.

Facultad de Economía, UADY (2011). Plan de Estudios de la Licenciatura en Economía.

Facultad de Economía, UADY. (2017). Historia. Recuperado de http://www.economia.uady.mx/index. php?item $=0$ \& cont $=0$ \&type $=0$ \&tema $=18$

Facultad de Economía, UADY. (2017). Misión. Recuperado de http://www.economia.uady.mx/index. php?item $=0 \&$ cont $=0$ \&type $=0 \&$ tema $=16$

Facultad de Economía, UADY. (2017). Visión. Recuperado de http://www.economia.uady.mx/index. php?item $=0$ \& cont $=0$ \&type $=0$ \&tema $=17$
Guilar, M E; (2009). Las ideas de Bruner: "de la revolución cognitiva" a la "revolución cultural". Educere, 13() 235241. Recuperado de http://www.redalyc.org/articulo. oa?id=3561457102

Reyes, G. E. (2007). Década de los 80: ajuste económico y crisis social. Zona económica. Recuperado de http://www. zonaeconomica.com/crisis-80

Santrock, J (2003). Psicología del desarrollo en la Adolescencia (3a.ed.). Capítulo 2 La ciencia del desarrollo adolescente. Capítulo 4 El desarrollo del pensamiento en los adolescentes. Madrid, España: Mc Graw-Hill/Interamericana de España,S. A. U.

Stabback, P. (2016). ¿Qué hace a un currículo de calidad?. Reflexiones en progreso $\mathrm{N}^{\circ} 2$ sobre Cuestiones fundamentales y actuales del currículo y el aprendizaje. Recuperado de http:/l unesdoc.unesco.org/images/0024/002439/243975s.pdf

UNESCO, (s.f). Planes y programas de estudios: análisis y revisión. El correo de la UNESCO. Recuperado de http:/l unesdoc.unesco.org/images/0013/001338/133817so.pdf 